\title{
Quantum phase transitions in matrix product systems
}

\author{
Michael M. Wolf ${ }^{1}$, Gerardo Ortiz ${ }^{2}$, F. Verstraete ${ }^{3}$ and J. Ignacio Cirac $^{1}$ \\ ${ }^{1}$ Max-Planck-Institute for Quantum Optics, Hans-Kopfermann-Str. 1, D-85748 Garching, Germany. \\ ${ }^{2}$ Los Alamos National Laboratory, Los Alamos, New Mexico 87545, USA. \\ ${ }^{3}$ Institute for Quantum Information, Caltech, Pasadena, US.
}

(Dated: February 2, 2008)

\begin{abstract}
We investigate quantum phase transitions (QPTs) in spin chain systems characterized by local Hamiltonians with matrix product ground states. We show how to theoretically engineer such QPT points between states with predetermined properties. While some of the characteristics of these transitions are familiar, like the appearance of singularities in the thermodynamic limit, diverging correlation length, and vanishing energy gap, others differ from the standard paradigm: In particular, the ground state energy remains analytic, and the entanglement entropy of a half-chain stays finite. Examples demonstrate that these kinds of transitions can occur at the triple point of 'conventional' QPTs.
\end{abstract}

A considerable part of modern condensed matter physics is devoted to the study of matter near zero temperature. In particular, zero-temperature quantum phase transitions (QPTs) [1], as observed in cuprate high-temperature superconductors and heavy fermion materials, have attracted enormous attention. Although an adaptation of the classical Landau-Ginzburg theory successfully describes some of these phase transitions, it is manifest that this concept is in general too narrow to cover all the fascinating aspects of the quantum world [2]. A complete and rigorous quantum mechanical description is, however, burdened by the notorious complexity of quantum correlations in highly entangled many-body systems.

The fields of condensed matter and quantum information theory study the behavior of quantum many-body systems by using complementary methodologies. Whereas the typical starting point in condensed matter theory is a Hamiltonian, from which states emerge as ground states (GSs) or excitations, quantum information theory deals primarily with quantum states, from which corresponding Hamiltonians may be constructed. For spin chains this point of view can be traced back to the seminal works on the AKLT model [3] and finitely correlated states [4], and it has recently been successfully resumed in various works on matrix product states (MPS) [5]. This led to new powerful numerical algorithms [6, 7, 8] accompanied by a better understanding of their efficiency [9], and new insights in renormalization group transformations [10] and sequential quantum generators [11]. Clearly, a fruitful crossfertilization is emerging between these two fields.

The present work investigates QPTs in systems represented by MPS by following the quantum information approach. It thus generalizes the findings of [4, 12] which already indicated the possibility of such transitions in MPS systems, and it enables us to theoretically design QPT's in quasi-exactly solvable models. In this way, we may engineer QPT points between phases whose correlations or symmetries we choose a priori. The corresponding orders can be of local type and/or of a more subtle hidden non-local character. The main observation behind is that, for the systems under consideration, a singularity in a $(\mathcal{D}-1)$-dimensional transfer operator leads to a QPT in the corresponding $\mathcal{D}$-dimensional quantum system. Although these findings hold for arbitrary $\mathcal{D}$, we focus on MPS in $\mathcal{D}=1$, where a general discussion is possible on full analytic grounds.

In fact, every state, in particular every GS, of a finite system can be represented as a MPS [4, 6]. The power of this representation - and with it the power of Density Matrix Renormalization Group (DMRG) - stems from the fact that in many cases a low-dimensional MPS already leads to a very good approximation of the state [9]. From such a low-dimensional MPS one can in turn construct a parent Hamiltonian from which it arises as an exact GS. We will study the dependence of correlation functions of such systems on a smoothly varying parameter $g$ and show that singularities can appear, which are reminiscent to those arising in known examples of QPTs. They appear only in the thermodynamic limit and are accompanied by diverging correlation lengths and vanishing energy gaps.

Some of the derived properties do, however, hardly fit within the conventional picture of QPTs in $\mathcal{D}=1$ spin systems: First, at the QPT point $g=g_{c}$ the GS energy density $e_{0}$ is analytic (Fig. 1). Typically, a non-analyticity in $e_{0}(g)$ is used as the defining property of a QPT [1]. We think, however, that the use of the term QPT is well justified by the presence of a non-analyticity of any observable quantity (e.g., two-point correlations). The second non-typical feature is the fact that the entropy of a half-chain remains finite as $g \rightarrow g_{c}$, which reflects the fact that MPS-QPTs cannot be described in terms of conformal field theory. In fact, a lot of attention has recently been devoted to the entanglement entropy [13, 14], resulting in the observation that the crossing of a QPT point typically coincides with the divergence of this entropy. The discussion below shows, however, that this is not the case for MPS QPTs in $\mathcal{D}=1$. A third peculiarity is, that although the breaking of a discrete symmetry can be engineered, MPS QPTs can occur without spontaneous symmetry breaking since for $g \neq g_{c}$ the GS is typically unique.

We will start by discussing the relevant properties of MPS and show how singularities can appear in the thermodynamic limit (size of the chain $N \rightarrow \infty$ ). Then, following the idea of [4] we show how the corresponding Hamiltonians can be constructed and discuss two examples, with local and nonlocal order, in greater detail. For one of these we show that the 
MPS-QPT occurs exactly at the triple point of 'conventional' QPTs.

Singularities in MPS - Consider a MPS which is up to normalization given by

$$
|\psi\rangle=\sum_{i_{1} \ldots i_{N}=1}^{d} \operatorname{tr}\left[A_{i_{1}} \ldots A_{i_{N}}\right]\left|i_{1} \ldots i_{N}\right\rangle,
$$

where $\left\{A_{i}\right\}$ is a set of $d D \times D$ matrices, $d$ is the Hilbert space dimension corresponding to one site in the chain and $D$ is the dimension of the bonds, when we think of the state in the valence bond picture. The state in Eq. (1) is translational invariant on a ring of length $N$, it has reflection symmetry if $A_{i}=A_{i}^{T}$, permutation symmetry if $\left[A_{i}, A_{j}\right]=0$ and time reversal symmetry if the $A_{i}$ 's are real. Also other local symmetries (e.g., $S U(2), \mathbb{Z}_{2}$ ) can be enforced by imposing appropriate constraints on the $A_{i}$ 's [4, 15].

In the following we will consider systems where the matrices $A_{i}$ depend on a single real parameter $g$. It is important to note that if the $A_{i}$ depend on $g$ in an analytic or continuous way, then so will its parent Hamiltonian which we construct below. Clearly, we could consider more general cases with several parameters $g_{1}, g_{2}, \ldots$.

Correlation functions for $m$ consecutive sites are given by

$$
\begin{aligned}
\left\langle S_{1} \ldots S_{m}\right\rangle & =\frac{\operatorname{tr}\left[E_{\mathbb{1}}^{N-m} E_{S_{1}} \ldots E_{S_{m}}\right]}{\operatorname{tr}\left[E_{\mathbb{1}}^{N}\right]}, \text { with } \\
E_{S} & =\sum_{i, j=1}^{d}\langle i|S| j\rangle A_{j} \otimes \bar{A}_{i} .
\end{aligned}
$$

Here $S_{i}$ is any observable acting on the $i^{\text {th }}$ site and the bar denotes complex conjugation. For simplicity we will focus on the generic case where the transfer operator $E_{\mathbb{1}}$ is diagonalizable and non-degenerate for $g \neq g_{c}$. Taking the thermodynamic limit $(N \rightarrow \infty)$ only the right $|r\rangle$ and left $|r\rangle$ eigenvectors of $E_{\mathbb{1}}$ 's largest eigenvalue $\nu_{1}$ survive in Eq. (2). With the normalization $\langle l \mid r\rangle=1$ this leads to

$$
\left\langle S_{1} \ldots S_{m}\right\rangle=\left\langle l\left|E_{S_{1}} \ldots E_{S_{m}}\right| r\right\rangle / \nu_{1}^{m} .
$$

Hence, if for some $g=g_{c}$ there is a level crossing in the largest eigenvalues of $E_{\mathbb{1}}$, then there will typically be a discontinuity in the correlation functions (or their derivatives), even though the $A_{i}$ 's and with them the $E$ 's are analytic in $g$. Needless to say that the same argumentation holds for every observable with finite support.

A trivial example showing that discontinuities of any order $n$ are possible is given by $(D=d=2)$

$$
A_{1}=\left(\begin{array}{cc}
1 & 0 \\
0 & 1+g
\end{array}\right), \quad A_{2}=\left(\begin{array}{cc}
g^{n} & 0 \\
0 & 0
\end{array}\right) .
$$

Here, all derivatives $\partial_{g}^{k}\left\langle S_{i} S_{i+1}\right\rangle$ of order $k<n$ will be continuous at $g=g_{c}=0$, whereas the $n^{\text {th }}$-order derivative e.g. of $\left\langle\sigma^{x}\right\rangle$ turns out to be discontinuous.
Let us now discuss the properties of a general MPS in the vicinity of a transition point $g_{c}$. The decay of two-point correlations $\left\langle S_{i} S_{i+l}\right\rangle$ can be obtained from Eq. (2) by setting $E_{S_{2}}=\ldots=E_{S_{m-1}}=E_{\mathbb{1}}$ with $m=l+1$ and exploiting the Jordan decomposition of the transfer operator. This leads to

$$
\left\langle S_{i} S_{i+l}\right\rangle-\left\langle S_{i}\right\rangle\left\langle S_{i+l}\right\rangle \sim\left|\frac{\nu_{2}}{\nu_{1}}\right|^{l-1},
$$

where $\nu_{2}$ is the second largest eigenvalue of the transfer operator. As the coupling strength approaches its QPT point value, $g \rightarrow g_{c}$, we get $\left|\nu_{2}\right| \rightarrow\left|\nu_{1}\right|$. Then, the correlation length $\xi=1 / \log \left|\nu_{1} / \nu_{2}\right|$ diverges and one obtains long-range correlations at the transition point. Note that despite the diverging correlation length there is no power-law decay at the transition point (which can be different for $\mathcal{D}>1[16]$ ).

Since a lot of attention has recently been devoted to the relation between criticality and the scaling of the entanglement entropy [13] we give now an explicit formula for the latter. In fact, in recent works QPT points seem to be intimately connected with a logarithmic diverging behavior of the entropy of a block of consecutive spins when considered as a function of the block size. However, this is not the case for the class of QPT's discussed in the present work. The entropy of an asymptotically large block of a MPS can be calculated exactly by exploiting the freedom in the $A_{i}$ 's in order to fix the gauge $\sum_{i} A_{i} A_{i}^{\dagger}=\mathbb{1}, \sum_{i} A_{i}^{\dagger} \varrho A_{i}=\varrho$, where $\varrho$ is a density matrix acting on $\mathbb{C}^{D}$. By the renormalization group arguments of [4, 10] the spectrum of a large block converges to the spectrum of $\varrho^{\otimes 2}$ such that the entropy becomes $2 S(\varrho)=-2 \operatorname{tr} \varrho \log _{2} \varrho$. In particular, the entropy never exceeds $2 \log D$, irrespective of how close the system is to a QPT point. This immediately implies that states exhibiting a diverging growth of the entanglement entropy cannot be described by MPS with finite $D$ (explaining the difficulties of DMRG for critical systems). Moreover, following [17] the absence of a logarithmic divergence implies that MPS-QPTs cannot be described in terms of conformal field theory (as already indicated by the absence of power-law decaying correlations).

Higher dimensions - Although our main focus is on onedimensional quantum systems $(\mathcal{D}=1)$ we briefly discuss in this interlude how to extend the presented ideas to higher dimensions.

Note that in the above $\mathcal{D}=1$ case the QPT is traced back to a level crossing in the operator $E_{\mathbb{1}}$, which acts only on a single site, i.e., in dimension $\mathcal{D}-1$. However, if the transfer operator itself has a spatial substructure, we are led to a QPT in a higher dimensional system. To be more specific consider a $\mathcal{D}$-dimensional cubic lattice of size $N_{1} \times \ldots \times N_{\mathcal{D}}$ with periodic boundary conditions. A generalization of 11 , the so-called projected entangled pair state (PEPS [18]), is then obtained by replacing the bi-linear forms $A_{i}$ by tensors of order $2 \mathcal{D}$, i.e., $A_{i}:\left(\mathbb{C}^{D}\right)^{\otimes 2 \mathcal{D}} \rightarrow \mathbb{C}$ and the matrix product by a tensor contraction according to the edges of the lattice. As we can interpret the $\mathcal{D}$-dimensional lattice as a chain of $\mathcal{D}-1$ dimensional systems we can introduce a transfer operator $E_{\mathbb{1}}^{\prime}$ for this chain by contracting all $\prod_{i=2}^{\mathcal{D}} N_{i}$ operators $E_{\mathbb{1}}$ 


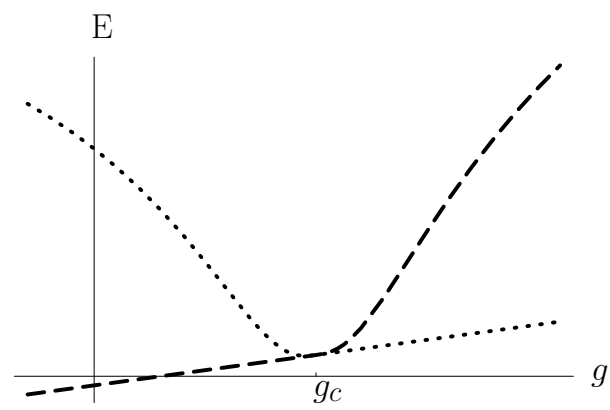

FIG. 1: Energies for the ground and first excited states as a function of $g$. In contrast to other QPTs the ground state energy of a MPS Hamiltonian remains (by construction) analytic at the QPT point $g=g_{c}$. Nevertheless the spectral gap vanishes and the correlation length diverges. Moreover, the non-analytic change of the ground state at $g_{c}$ is reflected by an observable non-analyticity of certain local expectation values.

on a $\mathcal{D}-1$ dimensional sub-lattice. In this way we are back to the one-dimensional scenario described above. That is, if we take $N_{1} \rightarrow \infty$ then a level-crossing in the largest eigenvalue of $E_{\mathbb{1}}^{\prime}$ can give rise to a QPT. Note that this way of constructing QPT points resembles the classical transfer matrix method discussed for instance in [19]. The problem of calculating the largest eigenvalue of $E_{\mathbb{1}}^{\prime}$ can numerically be tackled by DMRG (PEPS) algorithms for $\mathcal{D}=2(\mathcal{D} \geq 3)$. Particular instances of such transitions were discussed in [20], where power-law decaying correlations could be determined using Monte-Carlo methods. Note that by construction all critical ground states obtained in this way obey an area law for the entanglement entropy [14]. A method for deriving analytic results for particular higher dimensional instances will be provided elsewhere [16].

The Hamiltonians - Following the works on the AKLT model and finitely correlated states one can always construct a local Hamiltonian such that a given MPS is its GS: since the reduced state density operator $\rho_{k}$ corresponding to $k$ sites of a MPS has at most rank $D^{2}$, it has a nullspace whenever $k>$ $\log D^{2} / \log d$. Therefore, $|\psi\rangle$ is the GS of any Hamiltonian which is a sum of (local) positive operators supported in that nullspace. In particular, it is the GS of the Hamiltonian

$$
H=\sum_{i} \tau_{i}\left(P_{k}\right)
$$

with $P_{k}$ being the projector onto the nullspace of $\rho_{k}$ and $\tau_{i}$ its translation to site $i$. By construction the GS energy is always zero, i.e., it is evidently analytic in $g$.

Let us now see in which cases $H$ depends analytically on $g$ and, moreover, is such that $|\psi\rangle$ is its unique GS for $g \neq g_{c}$. Consider to this end the operator

$$
R=\mathcal{A}^{\otimes k}\left(\mathbb{1}_{D} \otimes \omega^{\otimes(k-1)} \otimes \mathbb{1}_{D}\right) \mathcal{A}^{\dagger \otimes k},
$$

where $\omega=\sum_{i, j=1}^{D}|i i\rangle\langle j j|$ and $\mathcal{A}|\alpha, \beta\rangle=\sum_{i}\left[A_{i}\right]_{\alpha, \beta}|i\rangle$. Note that, if $A_{i}$ depends analytically on $g, R$ also depends ana- lytically on $g$. It is evident from the valence-bond construction of the MPS that in general, $\operatorname{range}\left(\rho_{k}\right) \subseteq \operatorname{range}(R)$. However, if both eigenvectors $|r\rangle$ and $|l\rangle$ have full Schmidt rank, then a straightforward calculation shows that $\operatorname{range}\left(\rho_{k}\right)=$ range $(R)$. Hence, if this Schmidt rank condition is satisfied on both sides of $g_{c}$ (which is generically the case), then $H(g)$ indeed depends smoothly on $g$.

The uniqueness of the GS of Hamiltonians of the form (7) was discussed in [4, 15], where it was proven that it is unique if the largest eigenvalue of $E_{\mathbb{1}}$ is non-degenerate (i.e., $g \neq g_{c}$ ), $\operatorname{rank}\left(\rho_{k}\right)=D^{2}$ and

$$
\operatorname{range}\left(\rho_{k}\right) \otimes \mathbb{C}^{d} \cap \mathbb{C}^{d} \otimes \operatorname{range}\left(\rho_{k}\right)=\operatorname{range}\left(\rho_{k+1}\right) .
$$

It is also shown there that the latter condition is always satisfied if we replace $k$ by $k+1$, i.e., take $H=\sum_{i} \tau_{i}\left(P_{k+1}\right)$.

The analyticity of $H$ together with the uniqueness of its GS for $g \neq g_{c}$ immediately imply that a non-analyticity in the expectation values can only be caused by a vanishing energy gap at $g_{c}$.

Note that a degeneracy in the GS is equivalent to a degeneracy in $E_{\mathbb{1}}$. This means that there is no spontaneous symmetry breaking in one of the phases, unless $\left|\nu_{1}\right|=\left|\nu_{2}\right|$ for an entire interval (e.g., for $g \geq g_{c}$ ). However, for a degenerate $E_{\mathbb{1}}$ arbitrary broken discrete symmetries are possible [15].

Examples - After having discussed the general properties of MPS QPTs, we will consider some examples in more detail. Since the MPS have a very efficient parametrization in terms of the matrices $A_{i}$ we can, by imposing constraints on these matrices, engineer systems having desired properties (symmetries, orders, discrete symmetry breaking, etc.).

1. Three-body interactions: We start by considering the case $D=d=2$, i.e., $A_{1}, A_{2}$ being two-by-two matrices. By the arguments above every such state has a parent Hamiltonian with local three-body interactions. In fact, many of these Hamiltonians are similar to those appearing in (triangular) optical lattices [21, 22]. We construct an example with $\mathbb{Z}_{2}$ symmetry by imposing the existence of a similarity transformation which interchanges $A_{1}$ and $A_{2}$, i.e., $X^{-1} A_{1} X=A_{2}$ and $X^{-1} A_{2} X=A_{1}$. This is indeed the case if we choose

$A_{1}=\left(\begin{array}{ll}0 & 0 \\ 1 & 1\end{array}\right), A_{2}=\left(\begin{array}{ll}1 & g \\ 0 & 0\end{array}\right)$, and $X=\left(\begin{array}{ll}0 & g \\ 1 & 0\end{array}\right)$.

The corresponding transfer operator $E_{\mathbb{1}}$ has largest eigenvalues $1 \pm g$ leading to a singularity at $g=g_{c}=0$. A straightforward calculation shows a discontinuity in the first derivative of $\left\langle\sigma_{i}^{\alpha} \sigma_{i+1}^{\alpha}\right\rangle(\alpha=x, y, z)$, whereas all two-point expectation values are continuous. The magnetization in the $x$-direction can serve as an order-parameter since $\left\langle\sigma_{x}\right\rangle=4 g /(1+g)^{2}$ for $g>0$, whereas it vanishes for $g<0$.

At the QPT point $g_{c}$ the state is a GHZ state. For $g=-1$ (disordered phase) it is equal to the cluster state [23], and for $g=1$ (ordered phase) all spins point in the $x$-direction. 
The parent Hamiltonian is (by construction) $\mathbb{Z}_{2}$ symmetric:

$$
\begin{aligned}
H= & \sum_{i} 2\left(g^{2}-1\right) \sigma_{i}^{z} \sigma_{i+1}^{z}-(1+g)^{2} \sigma_{i}^{x} \\
& +(g-1)^{2} \sigma_{i}^{z} \sigma_{i+1}^{x} \sigma_{i+2}^{z} .
\end{aligned}
$$

Hence, it is a combination of an Ising interaction with transverse magnetic field (11), and a cluster state Hamiltonian (12). Since a constant term was omitted, the GS energy density is $e_{0}=-2\left(1+g^{2}\right)$, and one can readily check condition (9) implying that the GS is indeed unique for $g \neq g_{c}=0$.

The above Hamiltonian can be embedded into a twoparameter family

$$
\begin{aligned}
H(\gamma, h)=-\frac{1}{2} \sum_{i} & \frac{1+\gamma}{2} \sigma_{i}^{x}-\frac{1-\gamma}{2} \sigma_{i-1}^{z} \sigma_{i}^{x} \sigma_{i+1}^{z} \\
& +h \sigma_{i}^{z} \sigma_{i+1}^{z}
\end{aligned}
$$

which can be mapped onto a system of non-interacting Fermions by a standard Jordan-Wigner transformation. Moreover, $H(\gamma, h)$ can be mapped onto the XY-model via a duality transformation [24, 25]. This in turn exhibits $2^{\text {nd }}$ order QPTs on the lines $h= \pm 1$ and $\gamma=0$ for $h \in(-1,1)$. The path parameterized by $g$ is given by $\gamma^{2}+h^{2}=1$ (the disorder-line in the XY-model). Hence, the MPS transition occurs at the triple point of 'conventional' QPTs exhibiting algebraically decaying correlations and diverging entanglement entropies [24].

2. Two-body interactions: The previous example corresponded to the case of a local order parameter. Let us finally discuss an example with non-local string order. To this end, consider $D=2, d=3$, i.e., states of a spin one chain which are GSs of nearest-neighbor interactions. The most popular MPS in this class is certainly the GS of the spin1 AKLT model, which exhibits a hidden (string) order. In fact, this state can be embedded into a one-parameter family with MPS QPT. If we choose $\left\{A_{i}\right\}=\left\{-\sigma_{z}, \sigma^{-}, g \sigma^{+}\right\}$, then the transfer operator $E_{\mathbb{1}}$ has eigenvalues -1 and $1 \pm g^{2}$ leading to a diverging correlation length for $g \rightarrow g_{c}=0$. Moreover, the first derivative of $\left\langle S^{z}\right\rangle$ has a discontinuity at $g_{c}=0$, where $\left\langle S^{z}\right\rangle \rightarrow 1$, i.e., the state becomes ferromagnetic. For $g=2$ we get the AKLT state and for $g=-2$ a state, which is equivalent to the latter up to a local unitary transformation. For $g \rightarrow \pm \infty$ the GS becomes the Néel GHZ state $(|\uparrow \downarrow \uparrow \ldots\rangle+|\downarrow \uparrow \downarrow \ldots\rangle) / \sqrt{2}$. As already shown in [4] (with a different parametrization and for $g<0$ ) the corresponding Hamiltonian is rotationally symmetric in the XYplane, gapped with a non-degenerate GS (unless $g=g_{c}$ ) and has the form

$$
\begin{aligned}
H=\sum_{i} & \left(2+g^{2}\right) \vec{S}_{i} \vec{S}_{i+1}+2\left(\vec{S}_{i} \vec{S}_{i+1}\right)^{2} \\
& +2\left(4-g^{2}\right)\left(S_{i}^{z}\right)^{2}+(g+2)^{2}\left(S_{i}^{z} S_{i+1}^{z}\right)^{2} \\
& +g(g+2)\left\{S_{i}^{z} S_{i+1}^{z}, \vec{S}_{i} \vec{S}_{i+1}\right\}_{+} .
\end{aligned}
$$

Note that since $E_{\mathbb{1}}(g)=E_{\mathbb{1}}(-g)$ the GSs corresponding to $\pm g$ merely differ by local unitaries.
Conclusion - Matrix product states provide the perfect playground for investigating novel types of quantum phase transitions that do not fit in the traditional framework. We provided an example of such a QPT at the triple point of convential phase transitions, and it is easy to construct many more such transitions: given two predetermined MPS and associated Hamiltonians for which they are the unique ground states, we can always construct a one-parameter family of MPS interpolating between the two. If on the chosen path the transfer operator exhibits a level crossing in the largest eigenvalue, then the system undergoes a QPT.

Acknowledgements: The authors are grateful to the Benasque Center for Science, where parts of this work were developed, and thank D. Perez-Garcia for interesting discussions. We acknowledge support by the DFG (SFB 631).

[1] S. Sachdev, Quantum Phase Transitions (Cambridge Univ. Press, Cambridge, 1999).

[2] Q. Si, S. Rabello, K. Ingersent, J.L. Smith, Nature 413, 804 (2001); T. Senthil, A. Vishwanath, L. Balents, S. Sachdev, M.P.A. Fisher, Science 303, 1490 (2004); T. Senthil, cond-mat/0411275 (2004); T. Senthil, L. Balents, S. Sachdev, A. Vishwanath, M.P.A. Fisher, Phys. Rev. B 70, 144407 (2004).

[3] I. Affleck, T. Kennedy, E.H. Lieb, H. Tasaki, Commun. Math. Phys. 115, 477 (1988); I. Affleck, E.H. Lieb, T. Kennedy, H. Tasaki, Phys. Rev. Lett. 59, 799 (1987).

[4] M. Fannes, B. Nachtergaele, R.F. Werner, Commun. Math. Phys. 144, 443 (1992).

[5] A. Klümper, A. Schadschneider, J. Zittartz, J. Phys. A 24, L955 (1991); Z. Phys. B 87, 281 (1992).

[6] F. Verstraete, D. Porras, J.I. Cirac, Phys. Rev. Lett. 93, 227205 (2004).

[7] F. Verstraete, J.J. Garcia-Ripoll, J.I. Cirac Phys. Rev. Lett. 93, 207204 (2004).

[8] M. Zwolak, G. Vidal, Phys. Rev. Lett. 93, 207205 (2004); G. Vidal, Phys. Rev. Lett. 93, 040502 (2004).

[9] F. Verstraete, J.I. Cirac, cond-mat/0505140 (2005); T. J. Osborne, quant-ph/0508031, M. B. Hastings, cond-mat/0508554

[10] F. Verstraete, J.I. Cirac, J.I. Latorre, E. Rico, M.M. Wolf, Phys. Rev. Lett. 94, 140601 (2005).

[11] C. Schön, E. Solano, F. Verstraete, J.I. Cirac, M.M. Wolf, Phys. Rev. Lett. 95, 110503 (2005)

[12] A. Klümper, A. Schadschneider, J. Zittartz, Europhys. Lett. 24, 293 (1993).

[13] G. Vidal, J.I. Latorre, E. Rico, A. Kitaev, Phys. Rev. Lett. 90, 227902 (2003); G. Refael, J.E. Moore Phys. Rev. Lett. 93, 260602 (2004); J.P. Keating, F. Mezzadri, Phys. Rev. Lett. 94, 050501 (2005); V. Korepin, Phys. Rev. Lett. 92, 096402 (2004).

[14] M.B. Plenio, J. Eisert, J. Dreissig, M. Cramer, Phys. Rev. Lett. 94, 060503 (2005); M.M. Wolf, quant-ph/0503219(2005).

[15] B. Nachtergaele, Comm. Math. Phys. 175, 565 (1996).

[16] F. Verstraete, M.M. Wolf, D. Perez-Garcia, J. I. Cirac, t.b.p.

[17] P. Calabrese, J. Cardy, J. Stat. Mech. P06002 (2004).

[18] F. Verstraete, J.I. Cirac, cond-mat/0407066 (2004).

[19] M. Kac, in Fundamental Problems in Statistical Mechanics II, ed. E. G. D. Cohen (North-Holland, Amsterdam, 1968); J.A. Cuesta and A. Sanchez, J. Stat. Phys. 115, 869 (2004).

[20] H. Niggemann, A. Klümper, J. Zittartz, Z. Phys. B 104, 103 (1997); H. Niggemann, A. Klümper, J. Zittartz, Eur. Phys. J. B 
13, 15 (2000).

[21] J.K. Pachos, M.B. Plenio, Phys. Rev. Lett. 93, 056402 (2004); J.K. Pachos, E. Rico, Phys. Rev. A 70, 053620 (2004).

[22] Many other three-body examples can be found. For instance $A_{1}=\sigma^{x}, A_{2}=\sqrt{g}\left(\mathbb{1}-\sigma^{z}\right) / 2$ or $A_{1}=\sigma^{+}, A_{2}=\sigma^{-}+$ $\sqrt{g}\left(\mathbb{1}+\sigma^{z}\right) / 2$. The second example leads, for instance, to

$H=-\sum_{i} g\left(\sigma_{i}^{x}+\sigma_{i}^{x} \sigma_{i+1}^{z}+\sigma_{i}^{z} \sigma_{i+1}^{x}+\sigma_{i}^{z} \sigma_{i+1}^{x} \sigma_{i+2}^{z}\right)$

$$
+\left(1+2 g^{2}\right) \sigma_{i}^{z}-2 \sigma_{i}^{z} \sigma_{i+1}^{z}-\sigma_{i}^{z} \sigma_{i+1}^{z} \sigma_{i+2}^{z} .
$$

[23] R. Raussendorf, H.J. Briegel, Phys. Rev. Lett. 86, 5188 (2001); Phys. Rev. Lett. 86, 910 (2001).

[24] I. Peschel, J. Stat. Mech. P12005 (2004).

[25] The duality transform is $\sigma_{j}^{x} \mapsto \sigma_{j-1}^{x} \sigma_{j}^{x}, \sigma_{j}^{z} \sigma_{j+1}^{z} \mapsto \sigma_{j}^{z}$. 